Dig Surg 1995;12:86

\title{
Subject Index Vol. 12, No. 1, 1995
}

\section{Aflatoxin 7}

Autotransplantation 34 Chemoembolization 73 Chemotherapy 73 Cirrhosis 53, 79 Computed tomography 22 Diagnostic criteria 16 Drainage 34 Epidemiology 45 Etiology $45 \alpha$-Fetoprotein 79 Hepatic resection 34 Hepatitis B 79

- virus 7

C virus 7 Hepatocellular cancer 73

carcinoma 22, 53 Interventional radiology 65 Intraoperative ultrasound 40 Liver resection 40 transplantation 61 Magnetic resonance imaging 22 Major hepatectomy 53

Neoplasm 22

p53gene 7

Pathological prognostic indicators 16

Pathology 16

Percutaneous ethanol injection 65

Perioperative care 53

Pringle's maneuver 34

Prognosis 16

Radiation therapy 73

Recurrence 45

Regional chemotherapy 73

Screening 79

Segmentectomy 40

Split liver transplantation 61

Subsegmentectomy 40

Subtotal hepatectomy 45

Survival 45,61,65

Total hepatic vascular exclusion 34

Transcatheter arterial chemoembolization 65 Ultrasonography 79 Ultrasound 22 